\title{
Dialectical Learning In Information Systems
}

Adam Reed, (Email: Areed2@CalStateLA.edu), California State University, Los Angeles

\begin{abstract}
The classical dialectical (study partner) learning method of Greek and Hellenistic antiquity remains the traditional learning method of Jewish religious and cultural studies, and enjoys widespread informal use by students in some American universities. The cognitive advantages of dialectical study have led to its adoption as a powerful, general cognitive methodology, for example in Extreme Programming (Beck 1999.) This article describes the author's experience with formally assigned dialectical study in a variety of undergraduate and graduate courses in Information Systems at California State University, Los Angeles.
\end{abstract}

\section{DIALECTICAL LEARNING}

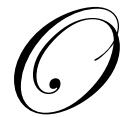

ur heritage from the classical Greek and Hellenistic civilizations includes not only specific works of philosophy, art, and science, but also a body of intellectual attitudes and methods of study. One of the most productive of these is the dialectical method of study and learning. The dialectical method is based on the realization that individuals have different perspectives grounded in different backgrounds and cognitive styles. By studying together with a dialectical partner, one gains an opportunity to consider interpretations, insights and solutions different from one's own. One also gains an opportunity to check one's understanding and extrapolations against the judgment of one's partner in study, whose different background and predispositions will often permit her to notice assumptions and errors that might have gone unnoticed when studying by oneself.

By limiting interactive involvement to a natural conversation between two (or at most three) participants, dialectical learning puts one in the intellectual focus of one's partner, a situation that does not permit inattention or dropping out of the discussion. In many cultures, learning and discussion takes place primarily in larger groups. While groups of four or more can often achieve a more refined consensus, in practice discussion in larger groups sometimes leads a few of the participants, particularly those who have, or think they have, less knowledge or experience than the rest, to refrain from contributing with their active participation to the work of the group, in the belief that the time of the group is better used attending to the contributions of more knowledgeable or more assertive participants. In current university courses, it is not unusual for one or two members of a team of four or more students to receive credit for the work of the team without individually achieving the level of mastery that the team project was supposed to demonstrate.

Dialectical learning is particularly effective in maximizing the development and contributions of the less advanced partner in the pair. In contrast with the expectation of an essentially one-way transfer of knowledge from master to student in the traditional lecture or question-and-answer formats, in dialectical interactions the more advanced partner - even a much more advanced partner - expects to learn, and benefits from learning, from inputs from the less advanced. In the words of Rabbi Hanina (founder of the Talmudic academy at Sepphoris and the man most likely responsible for the introduction of the Hellenistic method of dialectical learning into the study of the Talmud - still the traditional learning method of Jewish religious and cultural studies) "I have learned much from my teachers, from my colleagues even more, but from my students I have learned the most" (Hanina ca. 300 BCE.) Even when there is a significant disparity of academic level between members of the interacting pair, study partners both benefit from each other's contributions.

Informal dialectical learning arrangements are part of the student culture in the dormitories and other student living groups at some US universities, particularly those where a significant fraction of the incoming students are already experienced in learning together with a study partner. Where this culture flourishes, the existence and advantages of informal dialectical learning arrangements are assumed in the curriculum; the syllabi 
seldom mention it explicitly, but depend implicitly on the additional depth and breadth of understanding achieved by study partnerships. In recent years, these advantages have been applied to another cognitively demanding enterprise, that of software engineering.

\section{EXTREME PROGRAMMING}

The term "extreme programming" was introduced by Kent Beck (1999) in response to the failure of software engineering traditions based on rigid pre-planning and on engineering methodologies in which the over-all design, and the testing criteria, were pre-set and were deliberately made difficult to modify, even when errors in and potential improvements to the initial plan were evident to working programmers. Beck set out to create a software development methodology that not only allowed but welcomed principled change at every point in the software development cycle.

One danger of embracing change is that everyone is partial to his own ideas, and may mistake a change that merely conforms the software to his own cognitive style for a correction or improvement. The countermeasure, suggested by Beck's experience with dialectical learning, was to create programming pairs, analogous to study pairs, so that each partner's understanding of potential corrections and improvements was enhanced by the necessarily different intellectual perspective of his or her programming partner. In the "extreme programming" environment all coding and design is actually done in pairs; the partners scan and discuss their programming in real time, often sharing a single monitor and keyboard.

\section{EXTREME PROGRAMMING AND DIALECTICAL LEARNING AT CAL STATE LA}

When I first introduced "extreme programming" to Cal State LA Information Systems students, in my first year of teaching at this university, I used the analogy to dialectical study - and soon noticed that this analogy was not at all helpful to most of my students here. Only a small minority of Cal State LA students live in the university dormitories or other student living groups - we are a "commuter university," and most students live with their parents or, if they are older, in families of their own. While we occasionally get a student or two who are familiar with dialectical learning, in the absence of residential student communities there is no student culture through which familiarity with dialectical learning might spread to the rest. Most of our students come from communities in which consensus-forming groups or master-student relationships are expected, and in which dialectical learning relationships are not known or not assumed.

In the absence of a pre-existing informal culture of dialectical learning, I took the step of adding a study partnership requirement to the syllabi of courses, first in programming and software engineering courses and then in every course that I teach. I have required the use of study partnerships - normally pairs of students, with triples if there was an odd number of students in a course - in sophomore course in C++ and in Java; and in senior and graduate courses in data communication, local area networks, software engineering, advanced Java, Perl and the LAMP platform, Unix/Linux architecture and capabilities, Shell, server administration, and advanced Unix/Linux server administration. In nearly every course, students who follow instructions to discuss their readings and exercises with a study partner every week, from the beginning of the course, tend to do well throughout. About onethird of students without prior experience in studying with a partner ignore the requirement until the mid-term examination. If a student does less well than expected on the midterm, it usually turns out that the student missed the required dialectical learning sessions; most of these students start taking the requirement seriously at that point, and catch up, or nearly catch up, by the time they take the final examination in the course.

\section{IMPLEMENTATION}

The study partner method is implemented by including, in the course requirements specified in the course syllabus, a paragraph equivalent to the following: 
Study Partners: You are expected to select a study partner among your colleagues in the class (or two study partners, so that you will meet in a group of three). You will exchange telephone numbers and e-mail addresses among partners, and meet with your partner or partners each week to review your understanding of current course content. Please make sure to bring to class any issues that come up in reviewing matters with your study partner. If you miss any class work you are responsible for obtaining your study partner's notes and recollections, and for asking whatever questions you find necessary to fill out your understanding.

This paragraph is emphasized in the syllabus walk-through in the introductory lecture in each course. The students are expected to choose their partners during the mid-lecture break in the first lecture, and are polled after the lecture to make sure that everyone has a partner. Those that don't are helped in finding a study partner. Interactive surveys of how the study partnerships are functioning are included in the second and third lecture, and in the lecture after the mid-term examination, immediately following the examination wlk-through. Students are also asked, at the start of each lecture, for questions resulting from their most recent dialectical study sessions.

\section{ANCILLARY BENEFITS}

Apart from its cognitive advantages, the dialectical study requirement has significant logistical benefits that stem from having a study partner. Many students in our Information Systems programs, and especially those taking senior and graduate courses, are already working full-time in the field. There are also many with responsibilities for the care of children or of elderly or disabled family members. Sometimes these categories overlap. Those students are frequently "on call," and may need to leave the classroom at any time to deal with work or family emergencies. Sometimes they may not be able to attend a class to begin with. The study partner requirement includes an obligation for partners to take notes and ask questions on each other's behalf when the other is not in the class, and then to covey to the temporarily absent partner everything that took place in their absence.

Dialectical study also has the advantage of making the student aware, when she is unsure of her understanding of some point, but believes that she is the only one in the class with that quandary, that others - often including her study partner - also may not understand everything on the first attempt. This improves the quality of student feedback, so that difficult points can be identified and given additional attention following student feedback. And it increases the incidence of the teacher's greatest reward: creative questions that expand the teacher's own knowledge, or that lead to further, productive research.

\section{REFERENCES}

1. Beck, Kent. Extreme Programming Explained: Embrace Change. Reading, Massachusetts: AddisonWesley Professional, 1999.

2. Hanina bar Hana, Talmud (Taanit 7a.) Circa 300 BCE. 
NOTES 